Magnetic brain source imaging (MSI) of focal epileptic activity is reported in 455 cases of epilepsy examined preoperatively at University of Erlangen-Nuernberg, Germany (Stefan H et al. Brain 2003;126:2396-2405). The average sensitivity of magnetoencephalography (MEG) for specific epileptic activity was $70 \%$, whereas MSI identified the lobe to be resected in $89 \%$ of 131 patients who underwent surgery. Those with extratemporal epilepsies were identified in a higher percentage than temporal lobe cases. In a study of 20 patients with idiopathic generalized epilepsy compared to healthy controls, magnetic resonance spectroscopic imaging showed a progressive thalamic neuronal dysfunction, independent of the amount of spike and wave activity (Bernasconi A et al. Brain 2003;126:2447-2454). The complementary use of MEG and EEG was useful in localizing the origin of cortical myoclonus in the right temporo-occipital cortex in a 15 year-old girl with Lafora-body disease (Verrotti A et al. Acta Paediatr Oct 2003;92:12181222).

\title{
HEMISPHERECTOMY FOR INTRACTABLE EPILEPSY
}

Charts of 111 patients, ages 2 months to 20 years, who underwent hemidecortication for intractable unihemispheric epilepsy in the period 1975-2001 were reviewed at Johns Hopkins Hospital. The average time from seizure onset to operation was 3.6 years. Follow-up ranged from 3 months to 22 years ( 3 died in the perioperative period, 2 died later due to seizures, and 3 were lost to follow-up). Seizures were controlled in $65 \%, 21 \%$ had occasional seizures, and $14 \%$ were not benefited. One or no anticonvulsant was continued in $80 \%$, and $89 \%$ were ambulatory. Outcome was correlated with etiology; patients with seizures caused by migrational disorders were controlled in $51 \%$, whereas those with other seizure etiologies (Rasmussen and congenital vascular) were seizure-free in $71 \%(\mathrm{p}=0.05)$. (Kossoff $\mathrm{EH}$, Vining $\mathrm{EPG}$, Pillas DJ et al. Hemispherectomy for intractable unihemispheric epilepsy. Etiology vs outcome. Neurology October (1 of 2) 2003;61:887-890). (Reprints: Dr Eric Kossoff, Jefferson 128, The Johns Hopkins Hospital, 600 North Wolfe St, Baltimore, MD 21287).

COMMENT. The Johns Hopkins extensive series of hemispherectomies for intractable seizures demonstrates a beneficial response in $86 \%$ of cases, and the outcome is particularly favorable in patients with seizures due to Rasmussen syndrome and vascular disorders. The ideal time to operate remains undetermined. Significant morbidity or mortality occurred in $7 \%$.

\section{RASMUSSEN SYNDROME: RESPONSE TO THALIDOMIDE}

A 13-year-old girl with Rasmussen syndrome diagnosed at 5 years, and right-sided seizures refractory to various therapies, including hemispherectomy, responded to thalidomide and is reported from Belgrade, Yugoslavia. Initial control with carbamazepine, valproate and clonazepam was followed by relapse and a more extensive right hemiparesis. Despite resection of the cortical epileptogenic zones in the left hemisphere at 9 years of age, and trials of different anticonvulsants, acyclovir, g-globulin, iv methylprednisolone, oral prednisone, and plasma exchange, seizure frequency 